\title{
Valence-band-shape modification due to band coupling in strained quantum wells
}

\author{
L. Viña and L. Muñoz \\ Instituto de Ciencia de Materiales de Madrid, Consejo Superior de Investigaciones Científicas, Madrid, Spain \\ and Departamento de Física de Materiales, C-IV, Universidad Autonoma, Cantoblanco, 28049 Madrid, Spain \\ N. Mestres \\ Departamento de Física de Materiales, C-IV, Universidad Autonoma, Cantoblanco, 28049 Madrid, Spain
}

E. S. Koteles

GTE Laboratories Inc., 40 Sylvan Road, Waltham, Massachusetts 02254

A. Ghiti and E. P. O'Reilly

Department of Physics, University of Surrey, Guildford GU2 5XH, United Kingdom

D. C. Bertolet

Department of Chemical Engineering, University of Washington, Seattle, Washington 98195

K. M. Lau

Department of Electrical and Computer Engineering, University of Massachusetts, Amherst, Massachusetts 01003

(Received 1 February 1993)

\begin{abstract}
We have observed the strong dependence of the in-plane effective masses of the heavy-hole and lighthole valence bands in quantum wells on their coupling. The magnitude of this interaction, which is a function of the energy separation of the two bands, was tuned by adjusting the magnitude of tensile strain and well width in $\mathrm{GaAs}_{1-y} \mathrm{P}_{y} / \mathrm{Al}_{0.35} \mathrm{Ga}_{0.65} \mathrm{As}$ quantum wells. A distinct increase of both the heavy- and light-hole masses is observed when the bands merge at $\mathbf{k}=0$, consistent with theoretical predictions.
\end{abstract}

Due to the extreme importance of a precise knowledge of the band structure in low-dimensional systems, a considerable theoretical effort has been dedicated to the calculation of hole-subband dispersion relations in strained and unstrained quantum wells (QW's) in the last decade. $^{1-9}$ These calculations indicate a strong mixing between the heavy-hole (hh) and light-hole (lh) states at $\mathbf{k} \neq \mathbf{0}$ and evidence the dependence of the effective masses on uniaxial stress. They predict that the hole dispersions may become very sharp or flat at $\mathbf{k}=0$, depending on the magnitude of stress. A distinct variation of the $\mathrm{hh}$ effective mass with stress applied in the [001] direction has been calculated for $\mathrm{GaAs} / \mathrm{Ga}_{x} \mathrm{Al}_{1-x} \mathrm{As} \mathrm{QW}$ 's with a singularity occurring at $\sim 3$ kbar. $^{7}$ Nevertheless, experiments designed to probe the valence-band (VB) structure in QW's are much scarcer and limited to particular cases. Band mixing at finite wave vectors has been demonstrated in $n$-type modulation-doped QW's under uniaxial stress. ${ }^{10}$ The splitting of the VB maximum and the occurrence of the $\mathrm{lh}$ state as the highest confined valence state in systems under biaxial tensile strain have also been established. ${ }^{11,12}$ It has been also shown, from the temperature dependence of the Shubnikov-de Haas oscillations, that the highest subband in a biaxially compressed heterostructure can have a light-hole-like in-plane effective mass. $^{13}$

Optical absorption in high magnetic fields has been used in many bulk semiconductors to obtain basic parameters, such as hole effective masses and nonparabolicities.
Most of the information about the VB dispersion in twodimensional (2D) systems comes from magneto-optical measurements: a low zone-center effective mass in the highest hh subband and an electronlike zone-center mass for the highest lh subband have been confirmed by resonant Raman scattering ${ }^{14}$ and photoconductivity. ${ }^{15}$ Hole effective masses have been also deduced from photoluminescence excitation (PLE) experiments. ${ }^{16,17}$ Recent$1 y$, the dispersion curves of the hole states in QW's have been investigated in $p$-type double-barrier resonanttunneling structures. ${ }^{18}$ This study clearly reveals that some states correspond to a negative hole effective mass for in-plane motion.

In this paper we report on the changes in the hh- and lh-subband dispersion in strained QW's as a function of the energy separation of these subbands at $k=0$. It is well known that the electronic structure of III-V semiconductors can be conveniently modified by application of an external unaxial stress. The main effects of this perturbation, on the VB, are a lifting of the zone-center degeneracy of the $\mathrm{hh}$ and $\mathrm{lh}$ subbands and a deformation of the VB dispersion, which becomes highly anisotropic. ${ }^{10}$ Similar results are expected in strained layers as a consequence of the biaxial strain induced by the latticeparameter mismatch. The inclusion of strain gives an extra degree of freedom to tune the energy splitting between the $\mathrm{lh}$ and hh states, accessing a range of new subband structures, including the possibility of holes with electronlike zone-center masses and of the highest band 
being light-hole-like, thus allowing VB engineering and tailoring of the transport properties. ${ }^{19}$

We investigate $\mathrm{GaAs}_{1-y} \mathrm{P}_{y} / \mathrm{Al}_{0.35} \mathrm{Ga}_{0.65} \mathrm{As} \mathrm{QW}$ 's, grown on a GaAs substrate, by means of magneto-PLE measurements. This system represents a simple material choice to obtain tensile strain and therefore to tune the valence-subband separation. The presence of phosphorous in the well layer decreases the magnitude of the lattice parameter. This lattice mismatch is entirely accommodated in the $\mathrm{GaAs}_{1-y} \mathrm{P}_{y}$ as a biaxial tensile strain. $^{20}$ Previous PL (Ref. 20) and PLE (Ref. 12) data have been reported in this system demonstrating changes in the valence-subband separation for different $\mathbf{P}$ compositions. The observation of the diamagnetic shifts of the ground and excited states of the excitons, under the influence of a magnetic field, and the use of a 2D hydrogenic model $^{21}$ to fit the magnetointerband transitions is utilized to determine the reduced effective masses ( $m_{r}^{*}$ 's) of the excitons. ${ }^{17}$ From the $m_{r}^{*}$ 's we obtain the in-plane effective masses of the hole subbands, which can be used to monitor the changes in the valence-subband dispersion as the energy separation between $\mathrm{h} h$ and $\mathrm{lh}$ states is tuned by the strain and quantum confinement effects. A conspicuous flattening of the valence subbands is observed as the $\mathrm{hh}$ and $\mathrm{lh}$ confined levels approach each other. This observation constitutes, to the best of our knowledge, the first direct experimental evidence of the long-predicted enhancement of the VB effective masses when the hh and lh states are made to be degenerate at $\mathbf{k}=0$.

The samples were grown by organometallic chemicalvapor deposition on (100)-GaAs substrates. ${ }^{20}$ A GaAs buffer layer $(1 \mu \mathrm{m})$, and a $0.4-\mu \mathrm{m} \mathrm{Al}_{0.35} \mathrm{Ga}_{0.65} \mathrm{As}$ barrier layer, were followed by a single 120 - $\AA$-wide GaAs QW and a series of $\mathrm{GaAs}_{1-y} \mathbf{P}_{y} \quad \mathrm{QW}$ 's with different thicknesses, separated by $400-\AA \mathrm{Al}_{0.35} \mathrm{Ga}_{0.65} \mathrm{As}$ barriers. The $\mathbf{P}$ concentrations were determined by $\mathbf{x}$-ray diffraction measurements. ${ }^{12}$ In this work we will report on the results of QW's of 80 and $120 \AA$ and P concentrations of $5 \%, 8 \%$, and $12 \%$. 2-K PLE spectra were obtained with magnetic fields applied in the Faraday configuration up to $13.5 \mathrm{~T}$. The spectra were recorded with incident circularly polarized light. The emitted light was analyzed into its $\sigma^{+}$and $\sigma^{-}$components, and was detected with standard photon counting techniques.

The fan chart of the pseudoabsorption peaks obtained with $\sigma^{+}$polarization of the exciting light for a 120- $\AA$ $\mathrm{GaAs}_{0.95} \mathbf{P}_{0.05} / \mathrm{Al}_{0.35} \mathrm{Ga}_{0.65} \mathrm{As} \mathrm{QW}$ is depicted in Fig. 1 . In this quantum well the peak obtained at $0 \mathrm{~T}$ results from the overlap of the ground states heavy- $\left(h_{1 s}\right)$ and light-hole $\left(l_{1 s}\right)$ excitons; the larger oscillator strength of $h_{1 s}$ and the broadening of the peaks hinders the resolution of $l_{1 s}$ at $0 \mathrm{~T}$. As the magnetic field is increased two main effects are observed: $l_{1 s}$ becomes resolvable at $7 \mathrm{~T}$ and develops into a clearly resolved peak at $13.5 \mathrm{~T}$, and the $n s$-excited states become observable. The excited states appear in the region of the continuum, and their observation is favored by the reduction of the exciton radii and the increase in the binding energy with increasing H. The resolution of heavy and light excited states is achieved by an analysis of the polarization of the emitted

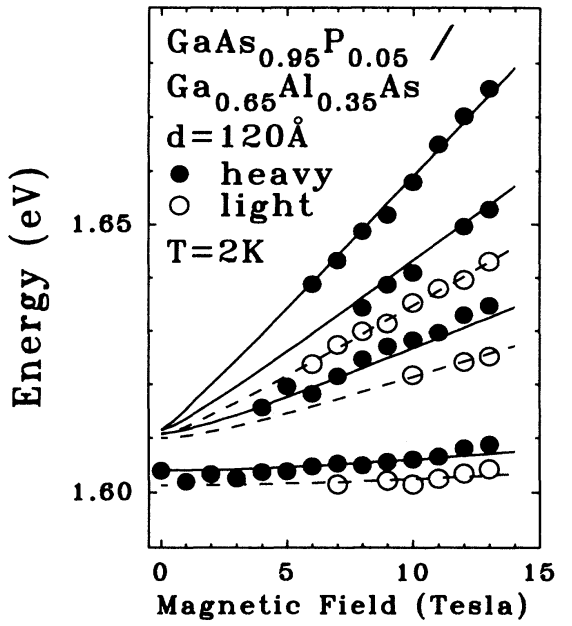

FIG. 1. Energies vs magnetic field of the ground- and excited-state heavy-hole (full circles) and light-hole (open circles) excitons for a $120-\AA \mathrm{QW}$. The solid (dashed) lines depict the results of the fits of the hh (lh) transitions with a $2 \mathrm{D}$ excitonic model (see text).

light. Two sets of transitions can be clearly distinguished. The ground and excited states of the heavyhole exciton, $h_{n s}(n=1-4)$, are shown as solid circles, while the corresponding light-hole excitons, $l_{n s}(n=1-3)$ are depicted as open circles. For the sample depicted in Fig. 1 the excited states of the $h \mathrm{~h}$ and $\mathrm{lh}$ excitons are accidentally degenerate at zero field, due to the compensation of strain and quantum confinement effects and the difference in binding energy of the excitons. At higher fields, the larger reduced effective mass of the lh exciton produces the splitting of the two fans.

The experimental results are fit using a 2D "hydrogeniclike" excitonic model, extending the analytical expressions given in Ref. 21 to the case of finite hole mass. The excitonic energies can be written as

$$
E_{n, m}=E_{g}^{2 \mathrm{D}}+\left(R_{y}^{*} / 4\right) \xi_{n, m}(z),
$$

where $E_{g}^{2 \mathrm{D}}$ is the energy gap, taking into account confinement and strain effects, $R_{y}^{*}$ is the effective Rydberg or binding energy, and $n, m$ are the principal and the angular quantum numbers, respectively. $\zeta_{n, m}(z)$ is a two-point Padé approximant, given by

$$
\zeta_{n, m}=\frac{P_{n, m}}{Q_{n, m}}=\frac{p_{n, m}^{(0)}+p_{n, m}^{(1)} z+p_{n, m}^{(2)} z^{2}+\cdots}{q_{n, m}^{(0)}+q_{n, m}^{(1)} z+q_{n, m}^{(2)} z^{2}+\cdots},
$$

where $z=\gamma^{1 / 2}$, and $\gamma$ is the dimensionless quantity $2 \hbar \omega_{c}^{r} / R_{y}^{*} . \omega_{c}^{r}=e H / m_{r}^{*}$ is the cyclotron frequency.

The coefficients $p_{n, m}$ and $q_{n, m}$ in Eq. (2) are taken from Table V of Ref. 21. Three parameters, $E_{g}^{2 \mathrm{D}}, R_{y}^{*}$, and $m_{r}^{*}$, were used to fit simultaneously the ground and excited states of the excitons. The hh and $\mathrm{lh}$ fans were fit separately, and the results of the fit are shown in Fig. 1 as solid and dashed lines, respectively.

The $m_{r}^{*}$ 's obtained from the fittings of the $h_{n s}$ and $l_{n s}$ excitons are shown in Table I, together with the energy splitting between $\mathrm{hh}$ and $\mathrm{lh}$ subbands, $\Delta=E_{g}^{2 \mathrm{D}}(\mathrm{lh})-E_{g}^{2 \mathrm{D}}(\mathrm{hh})$. These masses are a function of 
TABLE I. Reduced effective masses of the $\mathrm{hh}$ and $\mathrm{lh}$ excitons in $\mathrm{GaAs}_{1-y} \mathbf{P}_{y} / \mathrm{Ga}_{0.65} \mathrm{Al}_{0.35} \mathrm{As}_{\mathrm{QW}} \mathrm{QW}$ for different well thicknesses and phosphorus compositions. $\Delta$ is the subband separation between hh and lh states.

\begin{tabular}{|c|c|c|c|c|c|c|}
\hline \multirow{3}{*}{ P composition } & \multicolumn{3}{|c|}{$80 \AA$} & \multicolumn{3}{|c|}{$120 \AA$} \\
\hline & \multirow{2}{*}{$\begin{array}{c}\Delta \\
(\mathrm{meV})\end{array}$} & \multicolumn{2}{|c|}{$m_{r}^{*}\left(m_{0}\right)$} & \multirow{2}{*}{$\underset{(\mathrm{meV})}{\Delta}$} & \multicolumn{2}{|c|}{$m_{r}^{*}\left(m_{0}\right)$} \\
\hline & & hh & lh & & hh & lh \\
\hline $5 \%$ & 16.5 & 0.051 & 0.044 & -0.7 & 0.077 & 0.096 \\
\hline $8 \%$ & -0.9 & 0.060 & 0.094 & -12.4 & 0.063 & 0.068 \\
\hline $12 \%$ & 3.2 & 0.052 & 0.087 & -16.9 & 0.060 & 0.060 \\
\hline
\end{tabular}

the in-plane electron and hole effective masses $\left(m_{r}^{*-1}=m_{e}^{*-1}+m_{h}^{*-1}\right)$. The latter has a complicated dependence on $\mathrm{QW}$ thickness and $\mathrm{P}$ composition, due to the changes in the dispersion of the VB with confinement and strain. Therefore, no clear trend is observed in the $m_{r}^{*}$ masses varying these parameters.

To model our experiments, we have calculated the valence-subband dispersion as a function of the in-plane wave vector $\mathrm{k}$ using the $4 \times 4$ Lutinger-Kohn Hamiltonian in the axial approximation, with the calculation method described in Ref. 5. Since the Luttinger parameters are not well known for $\mathrm{GaAs}_{1-y} \mathbf{P}_{y}$, and we only try to predict the behavior of the VB dispersions in QW's as a function of $\Delta$, we have used the parameters of $\mathrm{GaAs} / \mathrm{Ga}_{0.65} \mathrm{Al}_{0.35} \mathrm{As} \mathrm{QW}$ 's. Figure 2 shows the VB dispersion relations for two different splittings $\Delta$ of the $\mathbf{k}=\mathbf{0}$ heavy- and light-hole states The model predicts a positive hh mass and a negative, electronlike, lh mass for $\Delta=11 \mathrm{meV}$ [Fig. 2(a)], which corresponds to the calculated difference in confinement for a $120-\AA$ unstrained QW. Important changes are expected in the in-plane effective masses as a result of the tensile strain: the splitting $\Delta$ decreases and therefore the effective mass of the hh should increase. ${ }^{19}$ We mimic the effects of strain in our model by keeping the $\mathbf{k}=\mathbf{0}$ eigenvalue for the hh subbands fixed while that for the lh subband is decreased with increasing biaxial tensile strain. The flattening of the hh subband and the change of curvature from electronlike to holelike of the lh subband are apparent in Fig. 2(b), when $\Delta$ is forced to be zero.

To investigate the changes in the VB dispersion relations, we have extracted the in-plane $\mathrm{hh}$ and $\mathrm{lh}$ effective

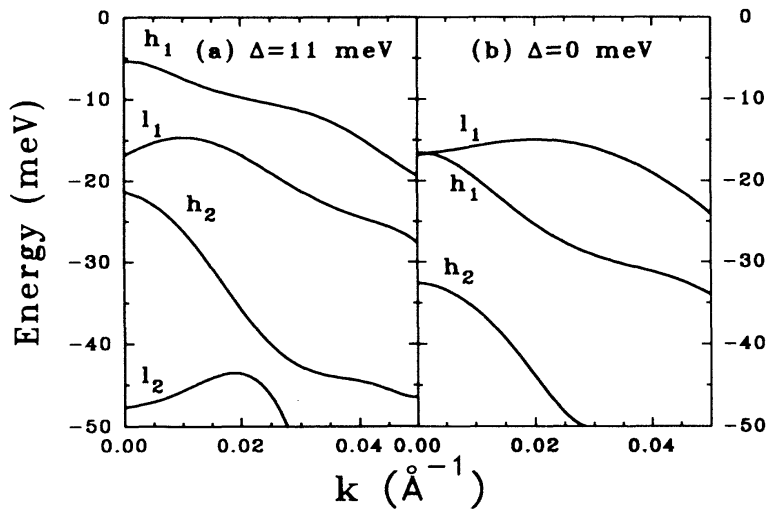

FIG. 2. Calculated VB dispersions in a three-band model for a heavy-light splitting $\Delta$ of (a) $\Delta=11 \mathrm{meV}$ and (b) $\Delta=0 \mathrm{meV}$. masses from the $m_{r}^{*}$ 's. The hole effective mass for the 2D excitons is the effective mass in the well plane averaged over a small region of $\mathbf{k}$ space near $\Gamma$. Thus this averaged effective mass will depend strongly on the band dispersion near $\Gamma$ and will reflect the changes in the VB structure. We have assumed a linear dependence of the electron effective mass on $y$ and used the values for GaAs and $\mathrm{GaP}$ from Ref. 22. We have obtained negative values of the in-plane effective masses as the bands become degenerate at $\mathbf{k}=0$. These derived values of the hole effective masses, especially the negative ones, must be treated with caution since their magnitude is determined from the difference of two similar quantities $\left[m_{h}^{*}=\left(m_{r}^{*-1}-m_{e}^{*-1}\right)^{-1}\right]$. The inaccuracies in the determination of $m_{r}^{*}$ can yield either very large positive or negative quantities when $m_{e}^{*} \sim m_{r}^{*}$. Actually a singularity will be obtained in the hole effective masses when the curvature of the bands changes from electronlike to holelike. Therefore we have plotted the inverse of the in-plane effective masses for the $\mathrm{h} h$ and $\mathrm{lh}$ subbands as a function of $\Delta$ in Figs. 3(a) and 3(b), respectively. ${ }^{23}$ The dashed lines depict the calculated inverse in-plane effective mass averaged from $\mathbf{k}=0$ to $\mathbf{k}=0.02$. A strong singularity is obtained in our model in the vicinity of $\Delta=0$ and the heavy- and light-hole masses change sign as the bands pass through each other due to the in-plane mixing of the $h h$ and $l h$ bands at small wave vector $\mathbf{k}$. It is seen that the hh mass is predicted and is found experi-

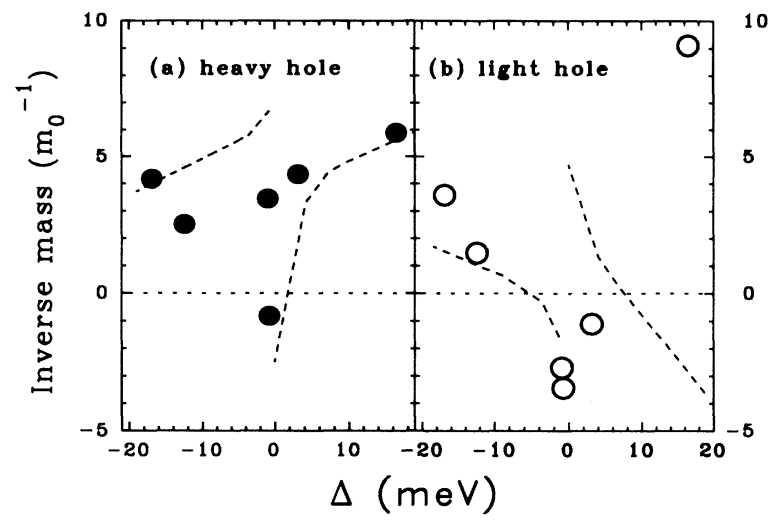

FIG. 3. Inverse of the in-plane hole effective masses as a function of the hh-lh subband splitting $\Delta$ for (a) heavy holes and (b) light holes, respectively. Dashed lines: calculated variation of in-plane mass with $\Delta$ for 120 - $\AA$ wells with $\Delta<0(80-\AA$ wells with $\Delta>0$ ). Filled circles: the experimental data for the samples shown in Table I. 
mentally to remain positive over the whole range of $\Delta$ values, except for small $\Delta$ when the interaction with the lh band gives an electronlike band dispersion (negative effective mass). Turning to Fig. 3(b), we find good agreement between theory and experiment when the light holes are at the valence-band maximum $(\Delta<0)$, with the mass being negative in both cases for small splittings. The agreement is less good for $\Delta>0$, particularly for the largest value of $\Delta$, where the inverse effective mass is predicted theoretically to be negative and is determined to be large and positive from experiment. The lh 1 state, however, interacts strongly in this case with both the hh1 and hh2 band and the experimentally measured mass may reflect the large positive value of the $\mathrm{hh} 2$ inverse effective mass in this case. In fact, the $\mathrm{hh}$ and $\mathrm{lh}$ states are strongly coupled in all of the cases considered and thus the excitons ${ }^{8}$ and the valence subbands ${ }^{1-4}$ have a mixed character, which will influence the values of our reduced effective masses. A more complete analysis taking these issues into account, such as that of Ref. 24, will probably yield better agreement between the theoretical and experimental results. In any case, the appearance of a singularity in the hole effective masses close to $\Delta=0$ in the calculations as well as in the experiments, demonstrates the strong modification of the VB dispersion relations in QW's obtained by tuning the subband separation.

In summary, we have determined the in-plane effective masses of the heavy- and light-hole subbands in tensilestrained QW's. We have found strong evidence of the flattening of the valence subbands as the energy difference between $\mathrm{hh}$ and $\mathrm{lh}$ states is decreased. This result confirms the predictions of band-structure calculations. It also has implications for devices. The valence-band structure that arises from biaxial tensile strain is particularly interesting for optoelectronic devices: with a proper choice of material parameters and quantum-well dimensions, the confined $\mathrm{hh}$ and $\mathrm{lh}$ excitons coincide. The overlap of both excitons and the increase of the hole effective masses will produce a larger absorption coefficient, ${ }^{20}$ which would improve the efficiency of photodiodes and high-speed optical modulators, and the saturation limit of heterostructure lasers.

We would like to thank G. Bastard and R. Ferreira for valuable discussions, and $\mathrm{H}$. Grant for a careful reading of the manuscript. This work was sponsored in part by CICYT Grant No. MAT-91-0201.
${ }^{1}$ A. Fasolino and M. Altarelli, in Two Dimensional Systems, Heterostructures and Superlatices, edited by G. Bauer, F. Kuchar, and H. Heinrich, Springer Series in Solid State Sciences Vol. 53 (Springer, Berlin, 1984), p. 176.

${ }^{2}$ U. Ekenberg and M. Altarelli, Phys. Rev. B 32, 3712 (1985).

${ }^{3}$ G. D. Sanders and Y. C. Chang, Phys. Rev. B 31, 6892 (1985).

${ }^{4}$ D. A. Broido and L. J. Sham, Phys. Rev. B 31, 888 (1985).

${ }^{5}$ W. Batty, U. Ekenberg, A. Ghiti, and E. P. O'Reilly, Semicond. Sci. Technol. 4, 904 (1989).

${ }^{6}$ G. Platero and M. Altarelli, Phys. Rev. B 36, 6591 (1987).

${ }^{7}$ J. Lee and M. O. Vassell, Phys. Rev. B 37, 8855 (1988); 37, 8861 (1988).

${ }^{8}$ G. W. E. Bauer and T. Ando, Phys. Rev. B 38, 6015 (1988).

${ }^{9}$ R. Ferreira and G. Bastard, Phys. Rev. B 43, 9687 (1991).

${ }^{10}$ R. Sooryakumar, A. Pinczuk, A. C. Gossard, D. S. Chemla, and L. J. Sham, Phys. Rev. Lett. 58, 1150 (1987).

${ }^{11}$ P. Voisin, C. Delalande, M. Voos, L. L. Chang, A. Segmuller, C. A. Chang, and L. Esaki, Phys. Rev. B 30, 2276 (1984).

${ }^{12}$ E. S. Koteles, D. A. Owens, D. C. Bertolet, J.-K. Hsu, and K. M. Lau, Surf. Sci. 228, 314 (1990).

${ }^{13}$ J. E. Schirber, I. J. Fritz, and L. R. Dawson, Appl. Phys. Lett. 46, 187 (1985).

${ }^{14}$ D. Heiman, A. Pinczuk, A. C. Gossard, A. Fasolino, and M. Altarelli, in Proceedings of the 18th International Conference on the Physics of Semiconductors, edited by O. Engstrom (World Scientific, Singapore, 1987), p. 617.

${ }^{15}$ D. C. Rogers, J. Singleton, R. J. Nicholas, C. T. Foxon, and K. Woodbridge, Phys. Rev. B 34, 4002 (1986).

16J. C. Maan, G. Belle, A. Fasolino, M. Altarelli, and K. Ploog, Phys. Rev. B 30, 2253 (1984).

${ }^{17}$ M. Potemski, L. Viña, G. E. W. Bauer, J. C. Maan, K. Ploog, and G. Weimann, Phys. Rev. B 43, 14704 (1991).

${ }^{18}$ R. K. Hayden, D. K. Maude, L. Eaves, E. C. Valadares, M. Henini, F. W. Sheard, O. H. Hughes, J. C. Portal, and L. Cury, Phys. Rev. Lett. 66, 1749 (1991).

${ }^{19}$ E. P. O'Reilly, Semicond. Sci. Technol. 4, 121 (1989).

${ }^{20}$ D. C. Bertolet, J.-K. Hsu, and K. M. Lau, Appl. Phys. Lett. 53, 2501 (1988).

${ }^{21}$ A. H. McDonald and D. D. Ritchie, Phys. Rev. B 33, 8336 (1986).

${ }^{22}$ S. Adachi, J. Appl. Phys. 53, 8775 (1982).

${ }^{23}$ In the calculations, we have used a $120-\AA$ (80- $\AA$ ) -wide QW for negative (positive) $\Delta$ 's, since this choice reproduces closely the experimentally found $\Delta$ 's. Qualitatively, the theoretical results are similar if the QW width is kept constant for the whole range of $\Delta$ 's.

${ }^{24}$ F. Ancilotto and A. Fasolino, Phys. Rev. B 38, 1788 (1988). 